\title{
Monolayers and Multilayers of Some Liquid Crystal Forming Compounds
}

\author{
H. DieP-QuAng and K. UEberReiter \\ Fritz-Haber-Institut der Max-Planck-Gesellschaft, \\ Faradayweg 4-6, D-1000 Berlin 33, Germany.
}

(Received September 5, 1980)

\begin{abstract}
Monolayers of liquid-crystal forming compounds: $p$-heptylphenyl $p$ hexanoyloxybenzoate and 2-methylbutyl- $p$-( $p$-hexyloxybenzoyloxybenzoate) were spread on the water surface of an automatic recording film balance. On compression, these monolayers collapsed to form successive multilayers. The multilayers were found to be two, three and four molecules thick by a comparison of the molecular surface area at the collapse point of the monolayer and at the multilayer transition points. On expansion, there was a stepwise reduction in the multilayers. A model of molecular orientation in the multilayers has been proposed. The liquid-crystalline layers investigated can serve as model of biological membranes.
\end{abstract}

KEY WORDS Film Balance / Monolayer / Multilayer / Liquid Crystal /

Biological Membranes /

In recent years, monolayers of amphiphilic molecules on air/water interface have been used as models for investigating biological membranes. Spread monolayers of components of biological membranes were compressed on Langmuir's type film balance with variation in composition, temperature and substrate. The resulting changes in recorded compression isotherm, i.e., monolayer state, gave information on molecular interaction in biological membranes. ${ }^{1-3}$ The use of monolayers as membrane models, however, has a basic shortcoming, in that biological membranes consist primarily of two adjacent layers of parallel oriented molecules as opposed to only one layer in the spread monolayers. Condensed spread monolayers collapse with further compression to form liquid lenses or microcrystallites, depending on the bulk state of spread compounds. It has not yet been possible to build and to detect multilayers of defined thickness by the film balance method. Bilayers or multilayers which can be built up from collapsed monolayers are more suitable as membrane models and are presented in this paper.

\section{EXPERIMENTAL}

Eventual multilayers on air/water interface are most likely built by surfactants which also form mesogenic bulk phases in the temperature range of experiments. The following liquid-crystal forming compounds (Figure 1) were found to yield the most interesting results:

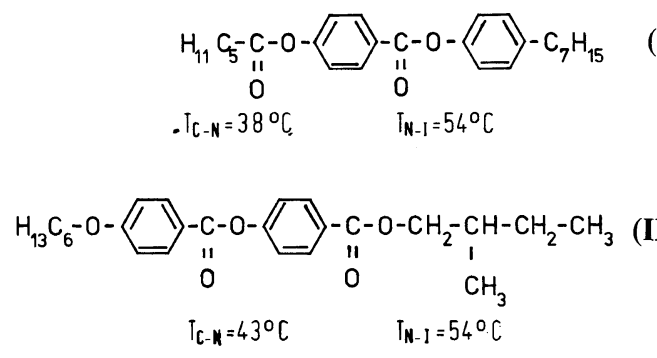

Figure 1. $p$-Heptylphenyl $p$-hexanoyloxybenzoate (I) 2-methylbutyl $p$-( $p$-hexyloxybenzoyloxybenzoate (II) $T_{\mathrm{C}-\mathrm{N}}=$ solid-nematic transition temperature. $T_{\mathrm{N}-\mathrm{I}}=$ nematic-isotropic transition temperature.

The compounds were purchased from Merck. Their purity was checked by DSC measurements. The film pressure/surface area isotherms were re- 
corded with a commercial automatic Lauda (Meßgeräte-Werk Lauda) film balance described elsewhere. ${ }^{4}$ The rate of compression was $24 \AA^{2}$ (molecule $\cdot$ minute) $)^{-1}$ for compound I and $3 \AA^{2}$ (molecule $\cdot$ minute $^{-1}$ ) for compound II. Variation in the compression rate did not produce any significant change in the shape of the recorded isotherms. Reproducibility of the curves was within 0.5 dyn $\mathrm{cm}^{-1}$ and $1 \AA^{2}$ molecule ${ }^{-1}$. Distilled water was used as the substrate. The temperature of the substrate and on air under the plexiglas cover of the film balance was kept constant within $0.1^{\circ} \mathrm{C}$. The investigated compounds and their spread monolayers were insoluble in water. There was no decrease in film pressure as long as the spread monolayers were kept at a constant surface area for 3 hours. The monolayers were spread on water from benzene solutions containing about $200 \times 10^{16}$ molecules per milliliter.

\section{RESULTS AND DISCUSSION}

Above $8^{\circ} \mathrm{C}$ (for compound I) and $20^{\circ} \mathrm{C}$ (for compound II) the isotherms exhibited a sharp break followed by a horizontal line (Figures 2 and 3). These isotherms were similar to those of monolayers which collapse on compression to produce liquid lenses since their bulk phase is in liquid state. ${ }^{5}$

On lowering the temperature, the plateau was interrupted by a break-down of the horizontal line. The resulting plateau became narrower until, with further lowering of temperature only a peak was left. This peak corresponds to the collapse of monolayers into solid bulk substances ${ }^{6}$ resulting in crystallization of monolayer material.

The gradual change of the plateau into a hump by lowering the temperature differs entirely from the usual pattern of isotherms of monolayers from substances whose melting points are within the range of the temperature variation. In the latter case, the horizontal line was replaced almost abruptly (at the melting temperature of bulk phase) with a hump. Figure 4 shows the difference between the isotherm pattern of compound II and that of elaidic acid.

The orientation of the spread molecules is horizontal due to the presence of two polar heads. If the monolayer of these molecules is compressed beyond the closest packing of horizontally oriented molecules, two different mechanisms can take place: 1) reorientation of the spread molecules by expulsion of one polar head out of water. With further compression, the spread molecules become vertically oriented. The position of the inflexion point, corresponding to the expulsion of one polar head out of water, changes strongly by change in temperature. $^{7}$ 2) The monolayer collapses by a "roll-over" mechanism of the horizontally oriented molecules. ${ }^{8}$

The inflection point (Figure 4a) does not change by variation of temperature so that one can assume a "roll-over" collapse mechanism for the monolayers investigated. For greater clarification this manner of reasoning is presented schematically in Figure 5.

Two-Dimensional Bilayered Crystallization Nucleus

The monolayer of compound II collapsed above $17^{\circ} \mathrm{C}$ like a liquid (horizontal line, Figure $4 \mathrm{a}$ ) and below $5.5^{\circ} \mathrm{C}$ like a solid (peak, Figure $4 \mathrm{a}$ ). Between $5.5^{\circ} \mathrm{C}$ and $17^{\circ} \mathrm{C}$, there was a combination of liquidlike collapse at point $L$ and crystallization at point $S$ (Figure 5). Crystallization of common collapsed monolayers is spontaneous and immediate below a certain temperature. The collapsed monolayer of liquid-crystalline compounds in contrast requires crystallization nuclei of sufficient size before this process can take place within a certain temperature region.

If one assumes a "roll-over" collapse (point L, Figure 5) for monolayers of rod-like, flat-lying molecules $^{8}$ these crystallization nuclei probably are bilayered structures of temperature-dependent critical size. If compression after collapse continues, the bilayered crystallization nuclei would grow gradually into a critical size. The collapsed monolayer would then crystallize spontaneously (point $\mathrm{S}$, Figure 5), causing a sharp drop in surface pressure to the value of the Equilibrium Spreading Pressure from the solid bulk phase.

In electron micrographs of monolayers of fatty acids, rupture lines can be seen at the moment of collapse. ${ }^{9}$ If the average number of disruption lines in the monolayers investigated remains the same for different temperatures, the width of the interrupted plateau may be considered as a scale for the size of the crystallization nucleus. In Figure 6, the width of the plateau of monolayer II $\left(\sigma_{\mathrm{L}}-\sigma_{\mathrm{S}}\right)$ is plotted against the corresponding temperature.

The size of a crystallization nucleus may cor- 
Monolayers and Multilayers of Some Liquid Crystal Forming Compounds
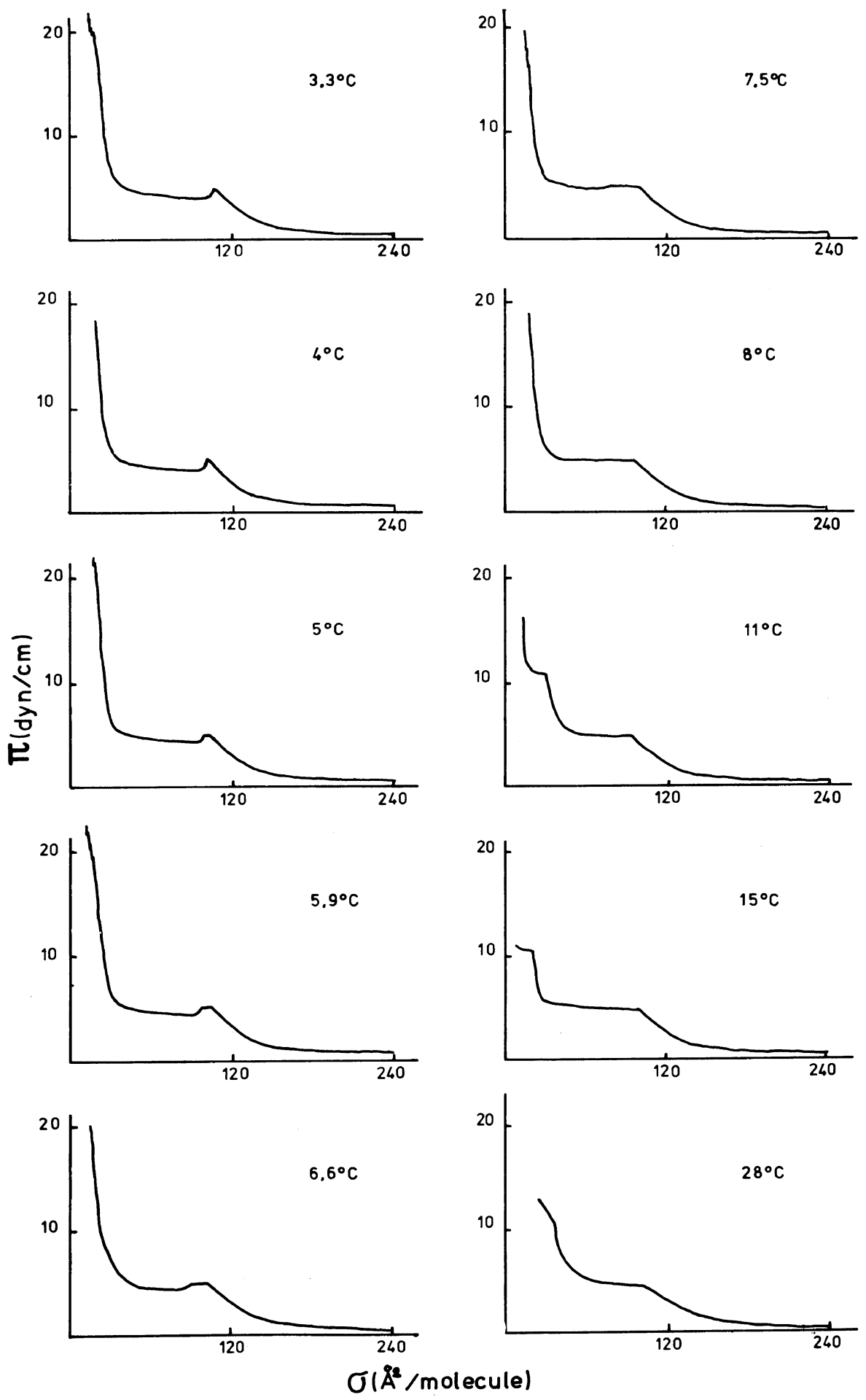

Figure 2. Isotherms of compound $\mathbf{I}$. 
H. Diep-Quang and K. UeberReiter
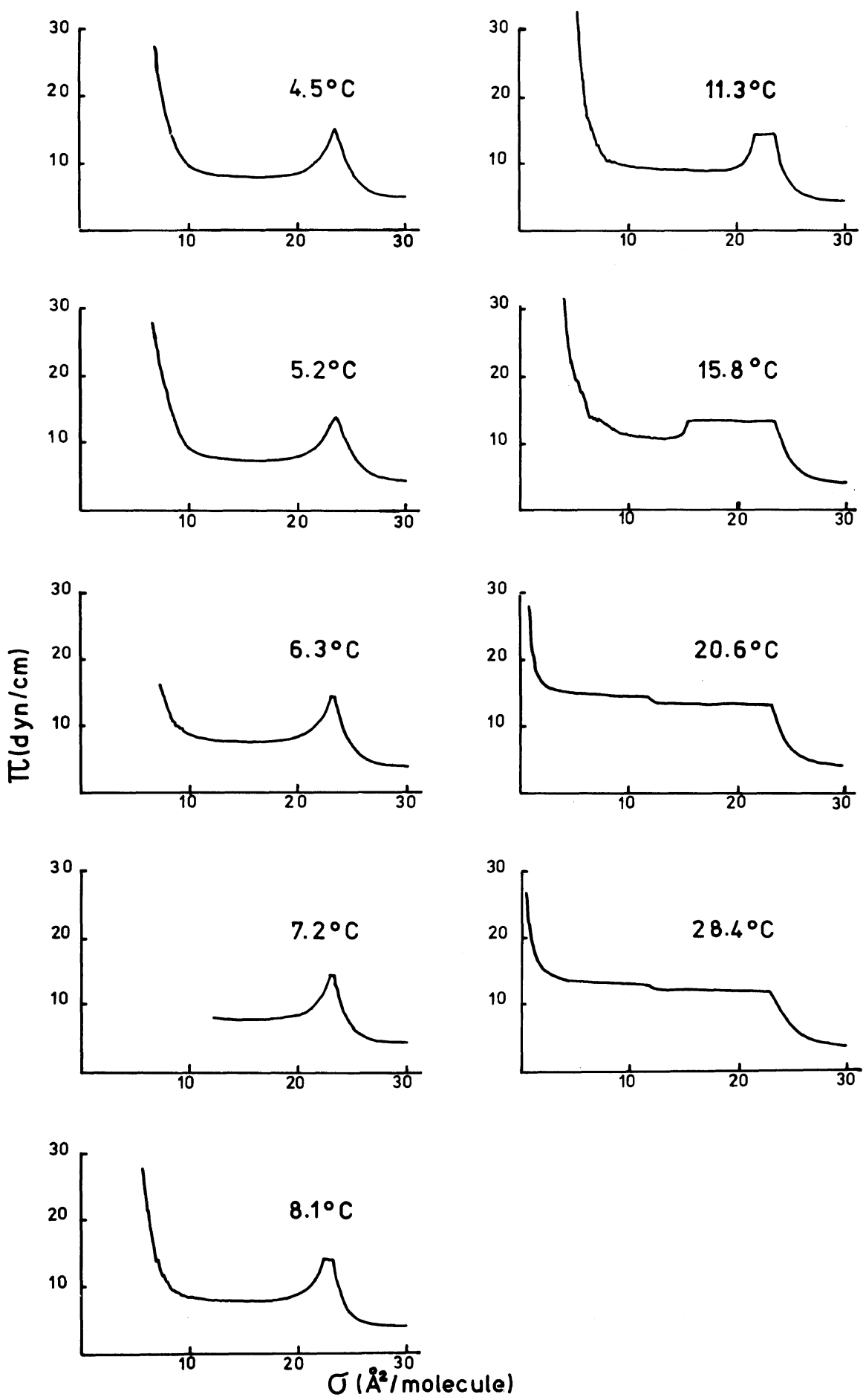

Figure 3. Isotherms of compound II. 

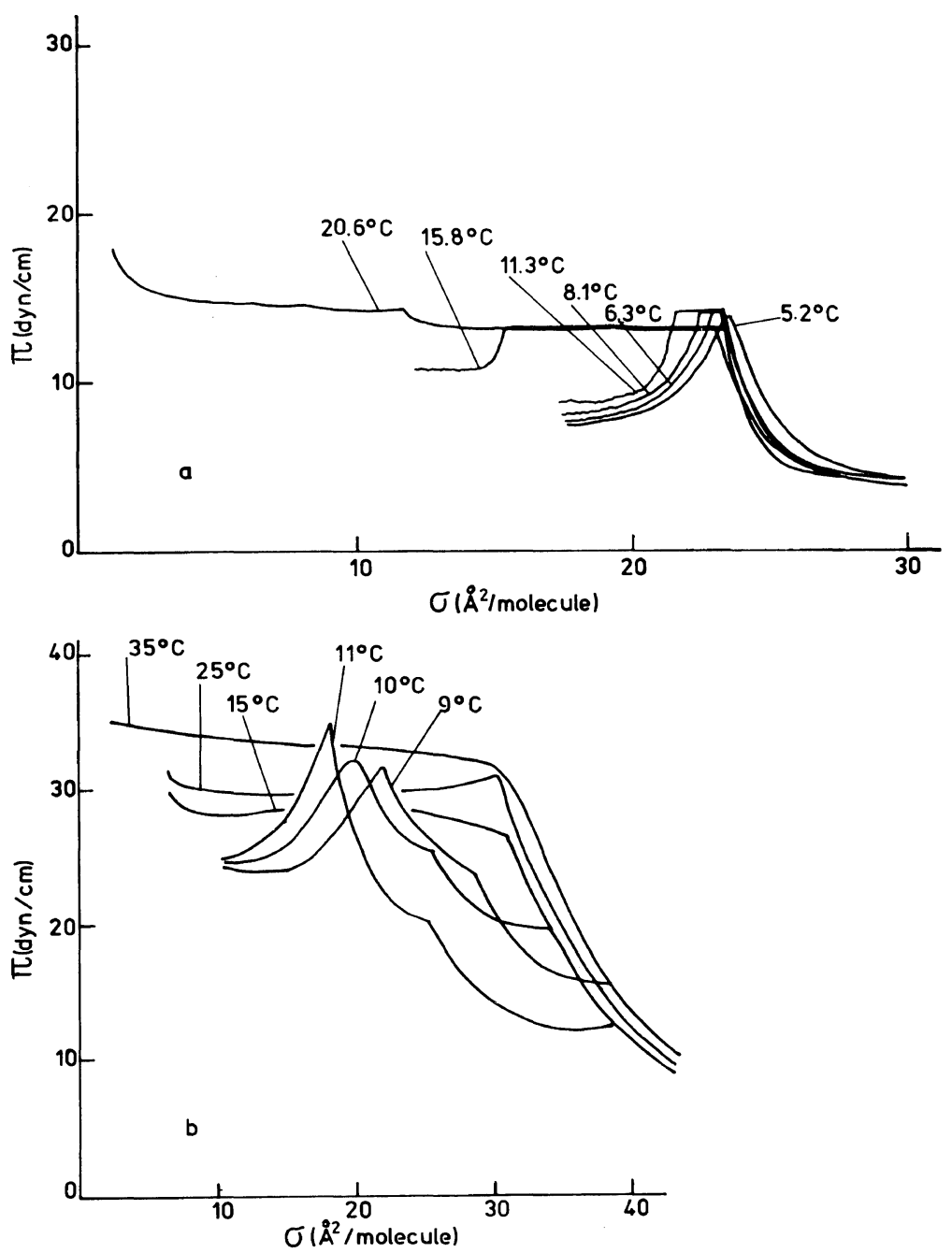

Figure 4. (a) Isotherms of compound II. (b) Isotherms of elaidic acid from Steinback and Sucker (H. Steinbach and C. Sucker, Colloid Polym. Sci., 255, 237 (1977)) reproduced by permission of Dr. Dietrich Steinkopf Publishing House.

respond to the cross section area of a swarm of liquid crystal molecules in the nematic phase, according to the swarm theory of nematic liquid crystals. ${ }^{10}$ In the surface film as a whole the molecules are randomly orientated but within the region of the crystallization nucleus, the orientation is parallel as in the bulk phase of nematic liquid crystals. Unfortunately, it is not possible to determine the number and size of the crystallization nuclei. But from Figure 7 , where $\sigma_{\mathrm{L}} / \sigma_{\mathrm{S}}$ is plotted against the temperature, one can guess that their average critical size grows with temperature until a limit is reached at $17^{\circ} \mathrm{C}$. At this temperature, the critical size of the crystallization nucleus coincides with the completion of the bilayer, as expressed by $\sigma_{\mathrm{L}} / \sigma_{\mathrm{S}}=2$.

The transition from the monolayer state to a crystallization nucleus must be reversible at constant temperature whereas the transition of a crystallization nucleus to the solid bulk phase must be irreversible. Thus, at equilibrium between the bilayer islands of smaller than critical crystallization nucleus size and spread molecules, a collapsed monolayer would return on expansion to the pre- 
Two mechanisms are possible for a closest packed monolayer of horizontal lying molecules with two polar groups:

a) reorientation of molecules by expulsion of one polar group from water; the molecules become vertically oriented on further compression or,

b) collapse of monolayer without reorientation of molecules.

a) reorientation of horizontal lying molecules at point $J(7)$. Collapse of monolayer at higher film pressure (point $\mathrm{S}$ ).

$T_{1}<T_{2}<T_{3}<T_{4}$
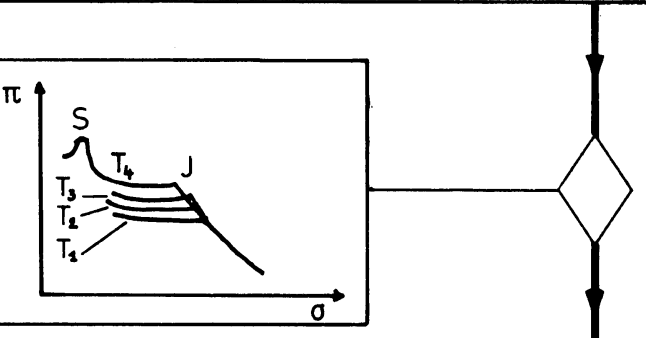

b) no reorientation on compression beyond the closest packing of horizontal lying molecules. The monolayer collapses with an "roll over" mechanism (8). Two forms of collapse are possible:

1) liquid-like collapse or,

2) crystal-like collapse.

1) the isotherm exhibits a horizontal line after liquid-like collapse (point L). The horizontal line changes into a hump (broken line) at melting temperature of bulk phase.

$\pi$

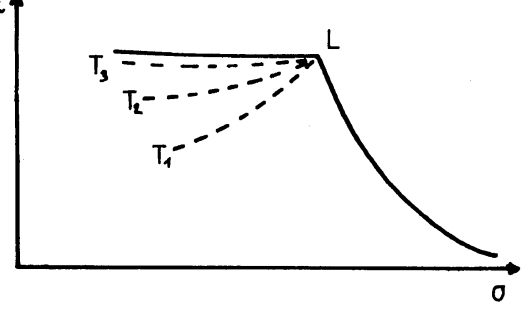

$T_{1}<T_{2}<T_{3}$
2) the isotherm exhibits a hump. Formation of microcrystallites after collapse of monolayer.

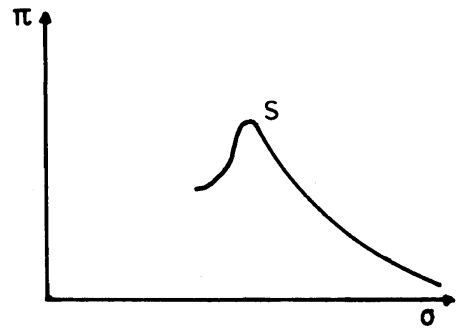

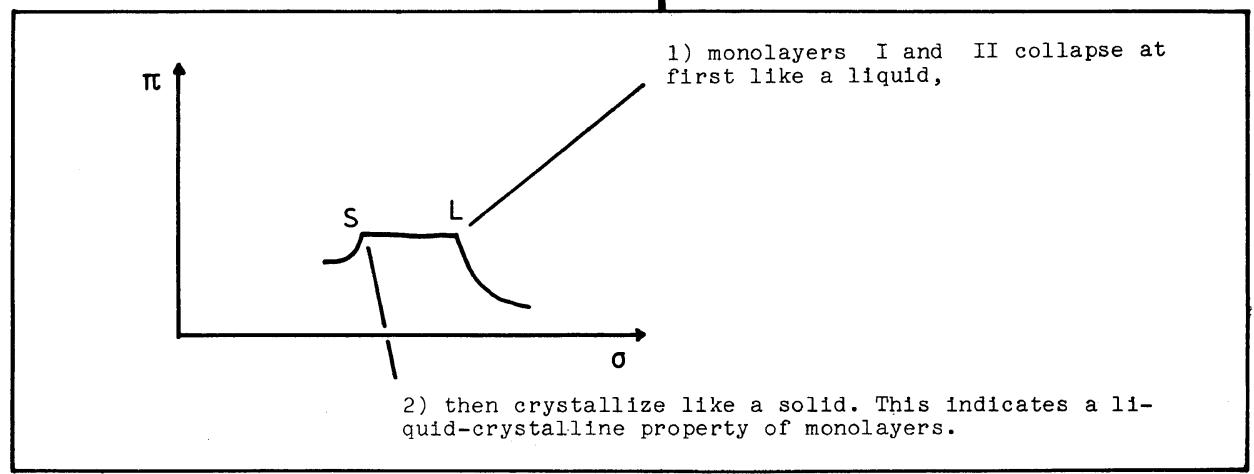

Figure 5. The used line of reasoning in schematized form. 


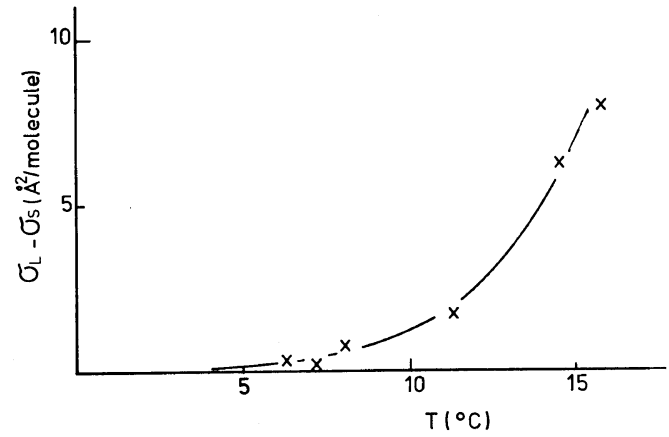

Figure 6.

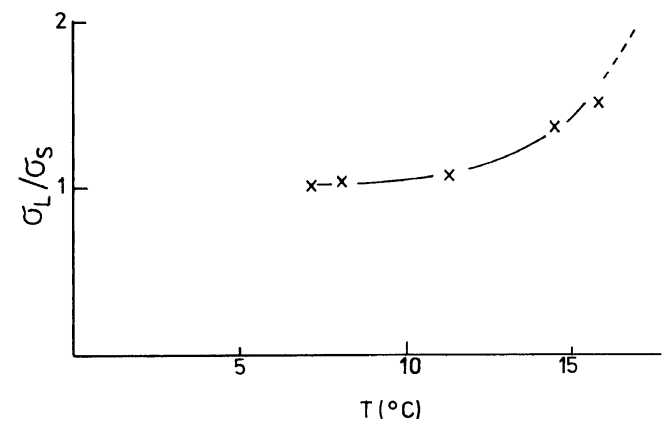

Figure 7.

vious state before collapse. On the other hand, a collapsed monolayer compressed beyond the point of crystallization cannot resume the previous state since an irreversible process - the crystallization has started.

To check this theory, the monolayer of compound II was successively compressed, expanded, and compressed again at $14.5^{\circ} \mathrm{C}$. At this temperature, the compression curve of monolayer II exhibited the typical interrupted plateau (Figures 3, 4a, and 5) between the collapse point $L$ and the crystallization point $S$. At the return point $R$, the direction of the moving barrier was reversed and the collapsed film expanded. This point $\mathrm{R}$ was placed alternatively between the points $\mathrm{L}$ and $\mathrm{S}$ in the middle of the plateau and beyond the crystallization point $\mathrm{S}$ where the surface pressure has dropped to the Equilibrium Spreading Pressure. These results are shown in Figure 8 and Figure 9.

The expansion of the collapsed monolayer after crystallization is shown in Figure 8. The surface pressure dropped almost to the two-dimensional vapour pressure immediately after the direction of

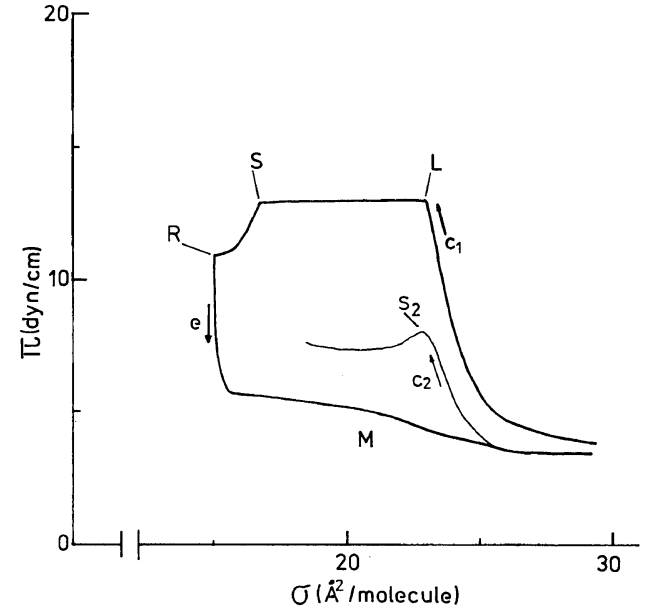

Figure 8. First curve $c_{1}$ : first compression, $L=$ collapse point; $\mathrm{S}=$ crystallization point. Second curve e: expansion, $\mathbf{R}=$ reversal point (beginning of expansion); $\mathbf{M}=$ transition from condensed to gaseous monolayer state. Third curve $\mathrm{c}_{2}$ : second compression, irreversible because of incompatibility with $\mathrm{c}_{1}$.

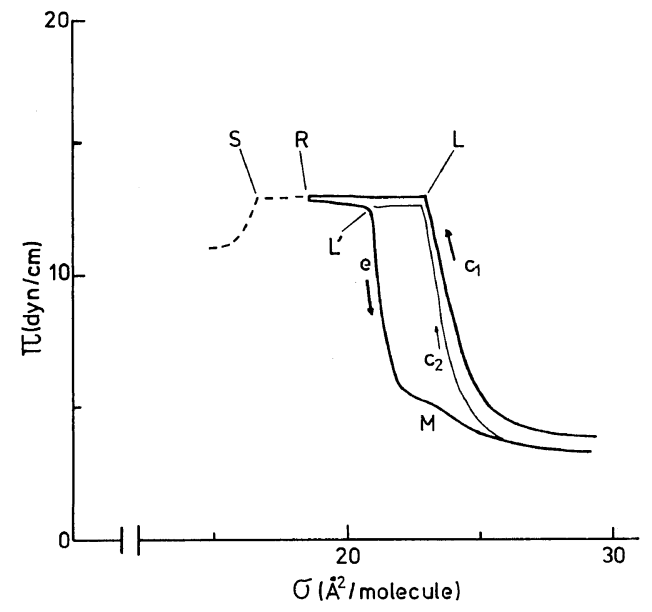

Figure 9. First curve $c_{1}$ : first compression, $L=$ collapse point; $\mathrm{R}=$ reversal point (beginning of expanison) prior to anticipated (broken line) crystallization point $\mathrm{S}$. Second curve e: expansion, $\mathrm{L}^{\prime}$ corresponding to $\mathrm{L}$; $\mathrm{M}=$ transition from condensed to gaseous monolayer state. Third curve $c_{2}$ : second compression, reversible because of congruity with $c_{1}$.

the moving barrier was reversed. At the second compression $\left(c_{2}\right)$, the collapse point $S_{2}$ was well below the collapse point of the first compression (L). It is remarkable that the curve of the second 


\section{H. Diep-Quang and K. UeberReiter}

compression $\left(\mathrm{c}_{2}\right)$ had a shape similar to isotherms of other common flat-lying molecules with two polar heads and high melting point. ${ }^{8}$ Obviously, the microcrystallites built up after point $\mathrm{S}$ remained in a metastable state, causing the premature collapse of the monolayer remnant at point $S_{2}$.

In Figure 9, the same procedure is demonstrated, except that the collapsed monolayer is not compressed until spontaneous crystallization occurs. Instead, the direction of the moving barrier is reversed as the anticipated middle of the plateau is reached. On expansion, the surface pressure does not drop immediately as in Figure 8, but remains nearly the same as on compression until point $L^{\prime}$. The rest of the expansion curve equals roughly the one in Figure 8.

The following second compression curve $c_{2}$ differs in a major way from Figure 8. Unlike the second compression curve shown in Figure 8, the $\mathrm{c}_{2}$ curve in Figure 9 is nearly the same as the first compression curve $c_{1}$. No crystallization process causes a major displacement of the $c_{2}$ curve towards smaller $\sigma$-values. No microcrystallite causes a premature collapse of the monolayer nor changes the liquid-like nature of the collapse.

This experiment proves that the state of the collapsed monolayer not compressed until the crystallization point $\mathrm{S}$ remains in equilibrium with the state of the monolayer before collapse. The transition between these states is reversible. This reversibility supports the theory of bilayered crystallization nuclei.

In the expansion curve e in Figure 9 the film pressure decreased sharply at point $L^{\prime}$ and smoothly at point $M$. The sharp drop in pressure at $L^{\prime}$ may be related to the relaxation effect on transition from bilayer to the monolayer and the smooth decrease in pressure at point $M$ can be attributed to the relaxation effect at the transition from monolayer liquid state to the monolayer vapour state.

\section{Formation of Multilayers}

a) Stepwise Build-up of Multilayers. At temperatures above $17^{\circ} \mathrm{C}$ the collapsed monolayer no longer can crystallize on the first compression (Figures 3 and $4 \mathrm{a}$ ). The theoretical area of bilayered crystallization nuclei then exceeds the area of available bilayer. This can be seen by the extrapolation of $\sigma_{\mathrm{L}} / \sigma_{\mathrm{S}}$ in Figure 7 to $17^{\circ} \mathrm{C}$. A limiting size of $\sigma_{\mathrm{L}} / \sigma_{\mathrm{S}}=2$ is reached at this temperature. This means

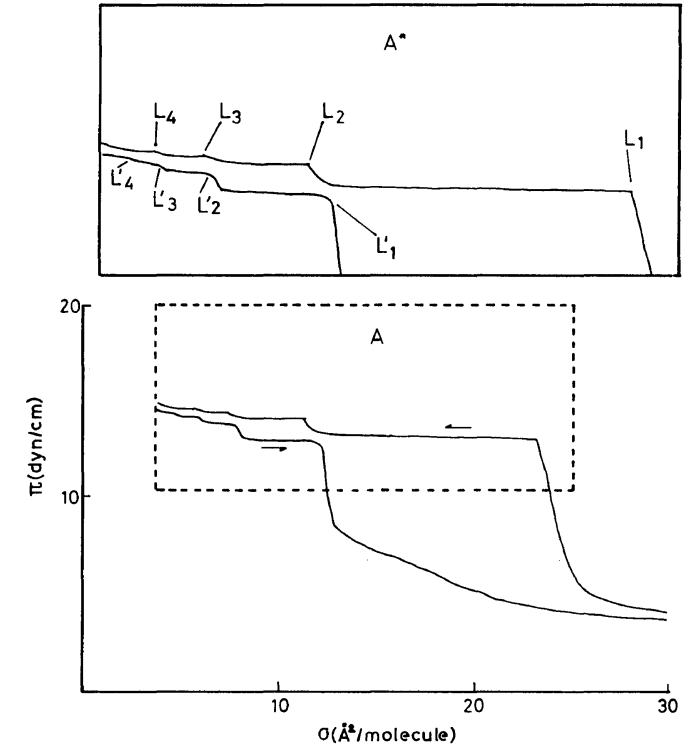

Figure 10. Stepwise elevation of the horizontal line at temperature above $17^{\prime \prime} \mathrm{C}$. Build-up of the second, third and fourth layer (from $L_{1}$ to $L_{4}$ ) on compression of compound II. Stepwise reduction of layers on expansion (from $\mathrm{L}_{1}{ }^{\prime}$ to $L_{4}{ }^{\prime}$ ) on expansion. $\mathrm{A}^{*}$ is the enlargement of area $\mathrm{A}$.

Table I.

\begin{tabular}{ccc}
\hline $\begin{array}{c}\text { Transition } \\
\text { points }\end{array}$ & $\begin{array}{c}\text { Molecular } \\
\text { limiting area }\end{array}$ & $\sigma_{1} / \sigma$ \\
\hline $\mathrm{L}_{1}$ & $\sigma_{1}=23.2 \AA^{2}$ molecule $^{-1}$ & $\sigma_{1} / \sigma_{1}=1$ \\
$\mathrm{~L}_{2}$ & $\sigma_{2}=11.4 \AA^{2}$ molecule $^{-1}$ & $\sigma_{1} / \sigma_{2}=2.03$ \\
$\mathrm{~L}_{3}$ & $\sigma_{3}=7.5 \AA^{2}$ molecule $^{-1}$ & $\sigma_{1} / \sigma_{3}=3.09$ \\
$\mathrm{~L}_{4}$ & $\sigma_{4}=5.7 \AA^{2}$ molecule $^{-1}$ & $\sigma_{1} / \sigma_{4}=4.07$ \\
\hline
\end{tabular}

that the whole layer is two molecules thick, $\sigma_{\mathrm{L}}$ being the limiting area for one molecule within the twodimensional closest packing of the monolayer before collapse.

Above $17^{\circ} \mathrm{C}$ the compression curve c clearly displays four transition points $\mathrm{L}_{1}, \mathrm{~L}_{2}, \mathrm{~L}_{3}$, and $\mathrm{L}_{4}$ (Figure 10). $\mathrm{L}_{4}$ is less spectacular, but still well discernible. The corresponding relaxation points $\mathrm{L}_{1}{ }^{\prime}, \mathrm{L}_{2}{ }^{\prime}, \mathrm{L}_{3}{ }^{\prime}$ and $L_{4}{ }^{\prime}$ can be seen on the expansion curve e. At point $L_{1}$, the molecules in the monolayer have the closest possible packing and $\sigma_{1}$ is limiting area of one molecule. To reveal the nature of the other transition points, it is interesting to form the quotients of $\sigma_{1}$ by $\sigma_{2}, \sigma_{3}$, and $\sigma_{4}$. These quotients 

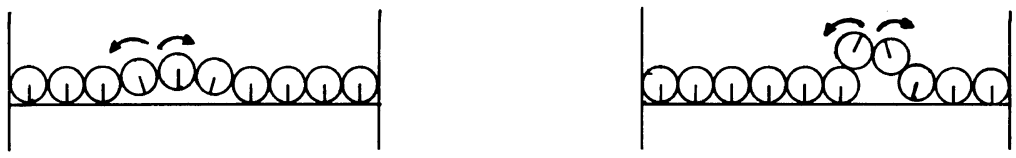

a

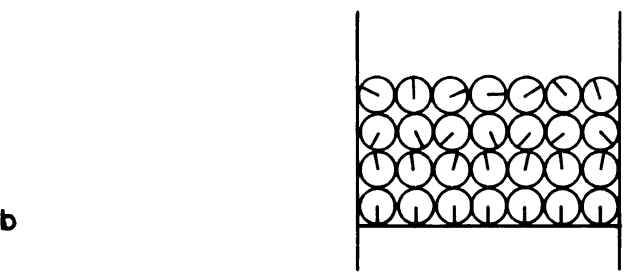

Figure 11. Cross section of molecules is presented by a circle with a notch indicating the orientation of polar groups: (a) "roll over" collapse; (b) orientation effect of interface on liquid-crystal molecules decreases rapidly from first to fourth layer.

were found to be almost exactly 2,3 , and 4 respectively, as shown in Table I:

At the transition point $\mathrm{L}_{2}$, the whole surface film was obviously 2 molecules thick since one molecule at this point occupied only half as much area as one molecule at the closest packing of the monolayer. By the same reasoning, it may be concluded that the film is three molecules thick at $\mathrm{L}_{3}$ and four molecules thick at $\mathrm{L}_{4}$ because $\sigma_{1} / \sigma_{3}=3$ and $\sigma_{1} / \sigma_{4}=4$.

From point $\mathrm{L}_{1}$ to nearly point $\mathrm{L}_{2}$, molecules from the collapsed monolayer were continuously transferred to the second layer as the film was further compressed. At point $\mathrm{L}_{2}$, the second layer was complete and the molecules within the bilayer were packed the closest. Continued compression caused the collapse of the bilayer. The same phenomena took place for the triple layer at point $\mathrm{L}_{3}$ and the quadruple layer at point $\mathrm{L}_{4}$.

During the time of this transfer of molecules from the lower layer to the upper layer, the film pressure remained constant as if an oil liquid was spread on water to build a duplex film. However, once the upper layer was completely occupied, more pressure was needed to break down the former multilayer structure and to create a new layer, as it happened at points $\mathrm{L}_{2}, \mathrm{~L}_{3}$, and $\mathrm{L}_{4}$. This stiffness of each multilayer at its completion requires some sort of long range order of the molecules within the layer. This long range order did not prevent the mobility of the molecules within each existing multilayer so that the pressure remained constant until completion of the upper layer. These facts fit well with the general properties of liquid crystals and are probably due to the mesogenic nature of the investigated compound.

As the number of layers increased, less excess pressure was needed to create a new layer:

$$
\pi_{4}-\pi_{3}<\pi_{3}-\pi_{2}<\pi_{2}-\pi_{1}<\pi_{1}-\pi_{\mathrm{e}}
$$

$\pi_{1}, \pi_{2}, \pi_{3}$ and $\pi_{4}$ are film pressures at points $L_{1}, L_{2}$, $\mathrm{L}_{3}$ and $\mathrm{L}_{4}$ respectively and $\pi_{\mathrm{e}}$, the Equilibrium Spreading Pressures. The excess pressure needed to create a new layer depends on the rotation barriers of the molecules on the long axis to "roll over" each other. ${ }^{8}$ Because of their polar heads, the flat lying molecules in the monolayer are orientated at the air/water interface and cannot rotate on the long axis. To overcome this interface orientation requires a very large increase in the film pressure, $\pi_{1}-\pi_{\mathrm{e}}$.

The influence of the interface on the molecules of the second layer is greatly reduced and the increase in film pressure at point $L_{2}$ is very small compared to the first increase at $L_{1}$. The influence of the interface on the molecules of the second layers is probably indirectly induced through orientation of the molecules of the first layer rather than being directly exerted by the interface itself. This induced orientation effect decreases rapidly for the third and the fourth layer. Since the molecules in these layers can rotate more freely along the long axis. The "roll 


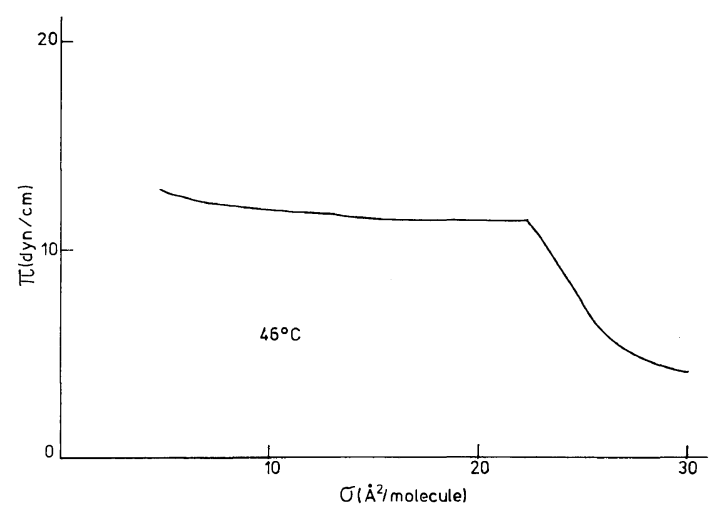

Figure 12. The monolayer of compound II collapses as an ordinary liquid at temperatures above the melting point of bulk phase.

over" mechanism for creating a new layer becomes almost as easy as that in a liquid bulk phase as indicated by the smooth shape of the transition points $L_{3}$ and $L_{4}$. A molecular model for the described monolayers and multilayers is proposed in Figure 11.

At a temperature of $46^{\circ} \mathrm{C}$, which lies between the solid-nematic and the nematic-liquid transition temperatures of the bulk phase, the long range arrangement of the molecules and the stiffness of the multilayers are destroyed. The compression isotherm at $46^{\circ} \mathrm{C}$ (Figure 12) shows no secondary transition points accordingly. The monolayer collapses in the manner of an ordinary liquid. b) Stepwise reduction of multilayers on expansion. The molecular limiting area at the transition points (on compression) is practically unaffected by variation in temperature as may be expected, since it results from the closest packed arrangement of molecules in the different layers. On the contrary, the corresponding relaxation points and the related hysteresis (on expansion) depend very much on the temperature as can be seen in Figure 13. At higher temperatures, the molecules in the closest packed layers have more energy for overcoming the intermolecular forces and to occupy the vacant air/ water interface created by expansion, thus reducing the relaxation time.

The sharp drop in pressure on relaxation from the different multilayer structures (points L) should be observed in contrast to the gradual inflexion at the transition from the condensed to the vapour monolayer state (point $\mathrm{M}$ ). This can be explained by

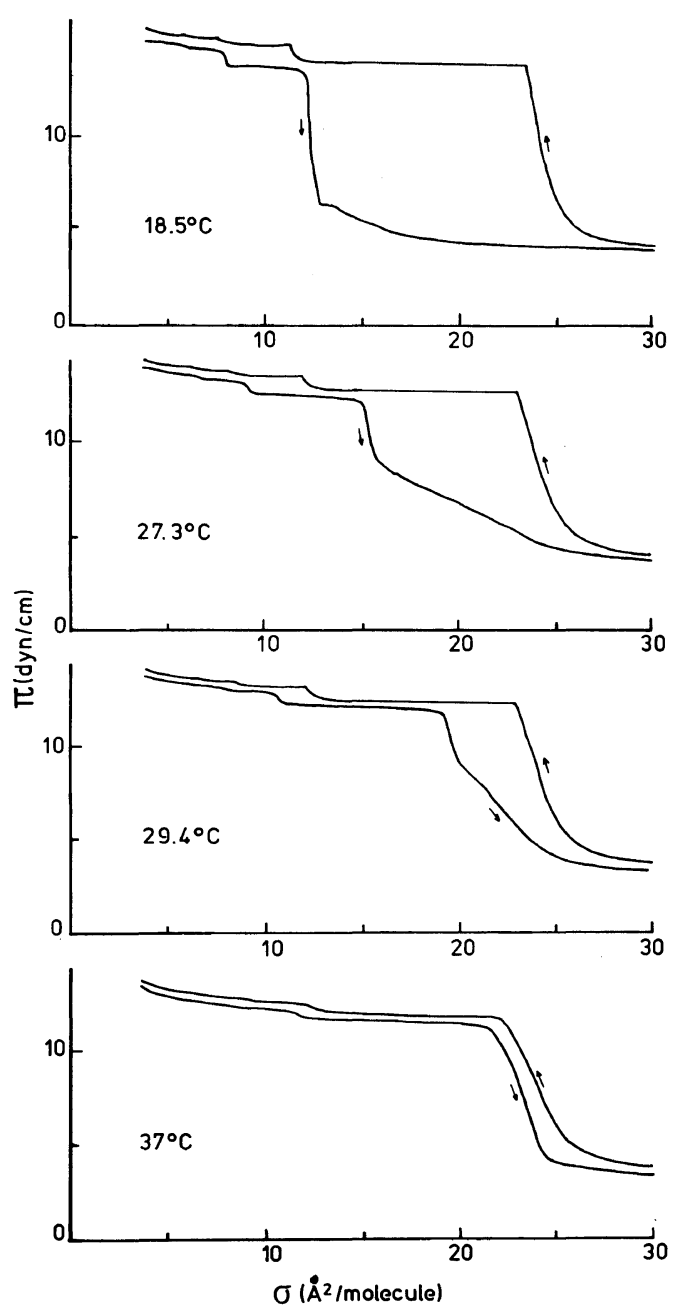

Figure 13. Temperature dependence of relaxation time on expansion of compound II. $\mathbf{M}$ is the transition point between the monolayer condensed and gaseous state.

the stronger intermolecular forces between the liquid-crystal molecules in the multilayer since the molecules in the upper layers are shielded from the adsorptive forces of the interface. On the other hand, the molecules in the condensed monolayer are also subjected to the competing attractive force between the water molecules and the hydrophilic groups of the liquid crystal molecules. This competing force enables the liquid-crystal molecules to be detached from the condensed monolayer islands, resulting in a gradual decrease in film pressure on expansion instead of a sharp drop. 


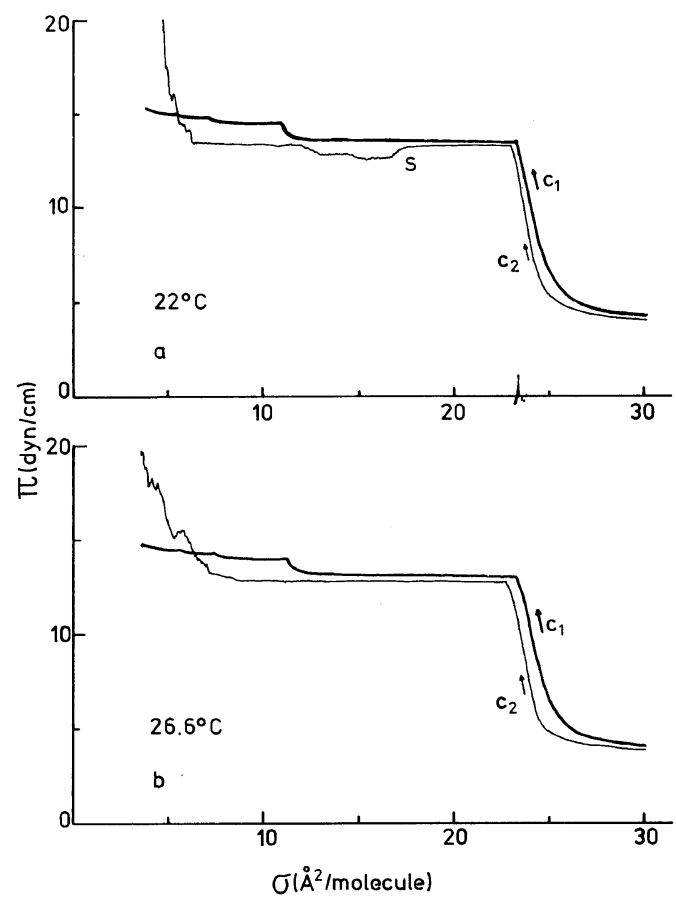

Figure 14. Stepwise build-up of multilayers of compound II cannot be repeated on second compression (curve $\mathrm{c}_{2}$ ).

The previously described stepwise formation of distinct and discrete multilayers could not be repeated with the second compression. Enough remnants of the different multilayers prevent the successive, stepwise formation of layers and cause an immediate crystallization into a solid bulk phase. The film pressure dropped and the plateau was interrupted on second compression because of premature crystallization (point S, Figure 14a). The film pressure could also raise irregularly since the floating microcrystallites were pressed against the recording membrane of the film balance in a small area (Figure 14b).

\section{CONCLUSION}

The very unusual combination of several physical properties in certain liquid crystal forming compounds enabled detection of the formation of successive, discrete and liquid-crystal-like multilayers on the air/water interface. A combination of these properties -thermotropic liquid-crystals, melting points in the temperature range of monolayer experiment, monolayer forming surfactants, flat-lying spread molecules owing to two polar heads - is very difficult to bring about and only a few compounds were found to have the required properties. However, new thermotropic liquid crystals are continuously discovered and the possibility exists that more compounds with the same characteristic properties can be spread on the air/water interface and compressed to form liquid-crystal-like multilayers. Such multilayers will provide interesting models for investigating membranes.

\section{REFERENCES}

1. P. Joos and R. A. Demel, Biochim. Biophys. Acta, 183, 447 (1969).

2. D. Chapman, N. F. Owens, M. C. Phillips, and D. A Walker, Biochem. Biophys. Acta, 183, 458 (1969).

3. M. Nakagaki and T. Handa, Bull. Chem. Soc. Jpn., 49, 880 (1976).

4. Chr. Sucker, Kolloid Z., 190, 146 (1963).

5. G. L. Gaines, in "Insoluble Monolayers at LiquidGas Interface," Wiley, New York, 1966, p 144.

6. H. E. Ries, Jr. and D. C. Walker, J. Colloid Sci., 16, 361 (1961).

7. B. M. J. Kellner and D. A. Cadenhead, J. Colloid Interface Sci., 63, 452 (1978).

8. P. W. Jeffers and J. Daen, J. Phys. Chem., 69, 2368 (1965).

9. H. E. Ries, Jr. and D. Walker, J. Colloid Interface Sci., 76, 361 (1961).

10. G. W. Gray, in "Molecular Structure and the Properties of Liquid Crystals," Academic Press, New York, 1962, p 66. 